ANNALES

POLONICI MATHEMATICI

LXVII.2 (1997)

\title{
On a method of determining supports of Thoma's characters of discrete groups
}

\author{
by ERNEst PŁONKA (Gliwice)
}

\begin{abstract}
We present a new approach to determining supports of extreme, normed by 1 , positive definite class functions of discrete groups, i.e. characters in the sense of E. Thoma [8]. Any character of a group produces a unitary representation and thus a von Neumann algebra of linear operators with finite normal trace. We use a theorem of H. Umegaki [9] on the uniqueness of conditional expectation in finite von Neumann algebras. Some applications and examples are given.
\end{abstract}

I. It is well known that any positive definite central function $\alpha$ of a group $G$ yields, via the Gelfand-Segal construction, a unitary representation $U^{\alpha}$ of $G$ in a separable Hilbert space and $\alpha$ can be extended to a finite, normal trace on the von Neumann algebra $\left\{U_{g}^{\alpha}: g \in G\right\}^{\prime \prime}$. Such functions form a compact convex set in the topology of pointwise convergence and correspond to the finite traces of the $C^{*}$-algebra of the group $G$. The extreme points of the sphere $\{\alpha: \alpha(1)=1\}$ are called characters in the sense of $E$. Thoma. If $\alpha$ is a character, the algebra $\left\{U_{g}^{\alpha}: g \in G\right\}^{\prime \prime}$ is a factor (cf. [8]). If $\{g \in G$ : $\alpha(g)=1\}=1$, the representation $U^{\alpha}$ is faithful and so $\alpha$ is called faithful. It follows from [1] that if $\alpha$ is 0 off some subgroup $H$ of $G$, then the representation $U^{\alpha}$ is the induced representation from the restriction $\alpha \mid H$ of $\alpha$ to $H$.

The problem of determining the supports of characters has been studied in many papers e.g. [2], [4], [5] and [6]. It has been proved in [5] that under some restrictions on $G$ each faithful character of a nilpotent group $G$ of class 3 is supported on the normal subgroup $G_{\mathrm{f}}$ of $G$ consisting of all elements with finite conjugacy classes. Such groups are called centrally inductive [2]. R. Howe [4] has shown that all finitely generated torsion free nilpotent groups are centrally inductive. In 1985 A. Carey and W. Moran [2] have established the same for countable nilpotent groups $G$ such that there exists an integer $n$ with the property that every finitely generated subgroup

1991 Mathematics Subject Classification: Primary 43A35; Secondary 43A40.

Key words and phrases: positive definite functions, characters, traces. 
of $G / G_{\mathrm{f}}$ is contained in a subgroup of $G / G_{\mathrm{f}}$ with $n$ generators. They have also proved this result for nilpotent complete groups, i.e. groups which contain all $n$th roots of their elements. The groups of unipotent upper triangular matrices with coefficients from a field of characteristic 0 are examples of such groups. Carey and Moran also gave an example of non-centrally inductive nilpotent groups. All the above papers do not use von Neumann algebras. We show how from a theorem of $\mathrm{H}$. Umegaki on conditional expectation in von Neumann algebras one can obtain a result which seems to be a useful tool in investigating the supports of characters.

Let $\mathbf{B}$ be a subalgebra of a von Neumann algebra $\mathbf{A}$ with a finite normal trace $\tau$. In [9], H. Umegaki proves that there is precisely one linear mapping $E: \mathbf{A} \rightarrow \mathbf{B}$ preserving the trace $\tau$ and the involution $*$ such that the equality

$$
E\left(b_{1} a b_{2}\right)=b_{1} E(a) b_{2}
$$

holds for all $a \in \mathbf{A}$ and $b_{1}, b_{2} \in \mathbf{B}$. The mapping $E$ is said to be the conditional expectation of $\mathbf{A}$ with respect to $\mathbf{B}$.

II. We start with a simple

LEMma. Let $\alpha$ be a *-preserving automorphism of a von Neumann algebra $\mathbf{A}$ with a finite, normal trace $\tau$ such that $\tau(\alpha(a))=\tau(a)$ for $a \in \mathbf{A}$. Let $\mathbf{B}$ be a subalgebra of $\mathbf{A}$, with $\alpha(b)=b$ for $b$ in $\mathbf{B}$. Then for the conditional expectation $\mathbf{E}$ of $\mathbf{A}$ with respect to $\mathbf{B}$ the mapping $E^{\alpha}$ defined by

$$
E^{\alpha}(a)=E\left(a^{\alpha}\right)
$$

is again a conditional expectation of $\mathbf{A}$ to $\mathbf{B}$ and consequently $E^{\alpha}=E$.

Proof. Straightforward.

THEOREM 1. Let $H$ be a normal subgroup of a group $G$ of unitaries in a separable Hilbert space such that the von Neumann algebra $\mathbf{A}=G^{\prime \prime}$ has a finite, normal trace $\tau$. Let $x$ be an element of $G$ such that $[x, g]=x^{-1} g^{-1} x g$ commutes with all $h$ in $H$ for $g \in G$. Assume also that $\left[x, h_{0}\right] \neq 1$ for some $h_{0} \in H$. If there is no non-trivial $G$-invariant projection in the von Neumann subalgebra $\mathbf{B}=\{1-[x, h]: h \in H\}^{\prime \prime}$ of the algebra $\mathbf{A}$, then $E(x)=0$.

Proof. The implementation mapping

$$
\mathbf{A} \ni a \rightarrow g^{-1} a g=a^{g} \in \mathbf{A}
$$

is an automorphism of $\mathbf{A}$ preserving $\tau$ and the involution $*$. Since the element $[x, g]$ commutes with all $h \in H$ for $g \in G$, we have, by the Lemma, $E^{h}=E$. Hence

$$
E^{h}(x)=E\left(h^{-1} x h\right)=E(x[x, h])=E(x)[x, h],
$$

which implies

$$
E(x)(1-[x, h])=0 \quad \text { for all } h \in H .
$$


This yields $E(x) P_{\mathrm{im}(1-[x, h])}=0$, where $P_{\mathrm{im}(b)}$ is the projection operator onto the image of the operator $b \in \mathbf{B}$. Since $\mathbf{B}$ is commutative, the projection $P_{\mathrm{im}(1-[x, h])}$ is the central support $c(1-[x, h])$ of the element $1-[x, h]$ (cf. [7]). Thus we have

$$
E(x) c(1-[x, h])=0 \quad \text { for all } h \in H,
$$

and consequently $E(x) P=0$, where $P=\operatorname{LUB}\{c(1-[x, h]): h \in H\}$.

Now the identity $[a b, c]=[a, c][[a, c], b][b, c]$ (cf. [3]) implies

$$
\left[x, h^{g}\right]=\left[x^{g}, h^{g}\right]=\left[x[x, g], h^{g}\right]=\left[x, h^{g}\right]\left[\left[x, h^{g}\right],[x, g]\right]\left[[x, g], h^{g}\right] .
$$

Since $h^{g},\left[x, h^{g}\right] \in H$ and $[x, g]$ commutes with $h \in H$, we obtain the equality

$$
[x, h]^{g}=\left[x, h^{g}\right] \quad \text { for all } h \in H \text { and } g \in G .
$$

Hence

$$
\begin{aligned}
P^{g} & =(\operatorname{LUB}\{c(1-[x, h]): h \in H\})^{g}=\operatorname{LUB}\left(\{c(1-[x, h]: h \in H\})^{g}\right. \\
& =\operatorname{LUB}\left\{(c(1-[x, h]))^{g}: h \in H\right\}=\operatorname{LUB}\left\{c(1-[x, h])^{g}: h \in H\right\} \\
& =\operatorname{LUB}\left\{c\left(1-\left[x, h^{g}\right]\right): h \in H\right\}=P
\end{aligned}
$$

for all $g \in G$. From our hypothesis it follows that the projection $P$ is not 0 , because there is an element $h_{0}$ in $H$ such that $1-\left[x, h_{0}\right] \neq 0$. Since $P$ is $G$-invariant, it has to be 1 projection. Hence $E(x)=0$, as required.

THEOREM 2. Let $\alpha$ be a positive definite function on a group $G$ such that $\alpha\left(x^{g}\right)=\alpha(x)$ for all $x, g \in G$ and $\alpha(1)=1$. Suppose that the restriction $\alpha \mid H$ of $\alpha$ to a normal subgroup $H$ of $G$ is a faithful, extreme point of the set $\left\{\beta: \beta(1)=1, \beta\left(h^{g}\right)=\beta(h), h \in H, g \in G\right\}$. Let $x \in G$ be an element such that $[x, g]$ and $h$ commute for all $h \in H, g \in G$ and $\left[x, h_{0}\right] \neq 1$ for some $h_{0} \in H$. Then $\alpha(x)=0$.

Proof. Let $U^{\alpha}$ be the representation of $G$ corresponding to $\alpha$ and $\mathbf{A}=$ $\left\{U_{g}^{\alpha}: g \in G\right\}^{\prime \prime}$ be the von Neumann algebra generated by $U_{g}^{\alpha}, g \in G$. Then $\alpha$ is (can be extended to) a finite, normal trace on $\mathbf{A}$. Let $\mathbf{B}=\left\{1-U_{[x, h]}^{\alpha}\right.$ : $h \in H\}^{\prime \prime}$. Since $\alpha$ is faithful and $1 \neq\left[x, h_{0}\right] \in H$, we have $U_{\left[x, h_{0}\right]}^{\alpha} \neq 1$ and, consequently, $\mathbf{B}$ is a non-trivial commutative subalgebra of $\mathbf{A}$. Moreover, since the restriction $\alpha \mid H$ is an extreme point of a $G$-invariant, positive definite function on $H$, there is no non-trivial $G$-invariant projection in $\mathbf{B}$ (Lemma 2 of [8]). Let $E$ be the conditional expectation of $\mathbf{A}$ with respect to $\mathbf{B}$. We see that all assumptions of the previous theorem are satisfied and therefore $E\left(U_{x}^{\alpha}\right)=0$. Since the mapping $E$ preserves the trace $\alpha$, it follows that $\alpha(x)=\alpha\left(U_{x}^{\alpha}\right)=\alpha\left(E\left(U_{x}^{\alpha}\right)\right)=\alpha(0)=0$, which completes the proof.

III. The following examples show how Theorem 2 can be used.

EXAMPLE 1. Let $R$ be a commutative, associative ring with identity $e$. The set $\left\{T_{a, b}: a, b \in R, a\right.$ invertible in $\left.R\right\}$ of all transformations $T_{a, b}$ : 
$R \rightarrow R$ defined by $T_{a, b}(x)=a x+b$ forms a group $G$ with multiplication $T_{a, b} T_{a^{\prime}, b^{\prime}}=T_{a a^{\prime}, a b^{\prime}+b}$. Obviously, $H=\left\{T_{e, b}: b \in R\right\}$ is a normal subgroup of $G$ with $[G, H]=H$. Thus the support of any faithful character of $G$ is contained in $H$.

Example 2. Let $\triangle_{k}, 0 \leq k \leq n$, be the set of all $n \times n$ matrices $A$ with entries from a commutative, associative ring with identity such that $A(i, j)=0$ for $i>j-k$. Let $\square_{k}, 0 \leq k \leq n$, be the set of all matrices $A \in \triangle_{1}$ with $A(i, j)=0$ for $i>n-k$ or $j \leq k$. It is clear that $\triangle_{k} \square_{n-k}=0$ for $k=0,1, \ldots, n$ and $\triangle_{k} \triangle_{l} \subseteq \triangle_{s}$, where $s=k+l(\bmod n)$. Hence $\left(\triangle_{k}\right)^{n}=0$ and therefore $G_{k}=\left\{I+x: x \in \triangle_{k}\right\}, 1 \leq k \leq n$, form a group with $(I+x)^{-1}=I-x+x^{2}-\ldots+(-x)^{n-1}$. Thus $\left[G_{k}, G_{1}\right] \subseteq G_{k+1}$ for $k=1, \ldots, n-1$. Observe that the element $I+x$ belongs to the centralizer $C_{G_{1}}\left(G_{k}\right)$ of $G_{k}, 1 \leq k \leq n$, in $G_{1}$ iff $x E_{r, s}=E_{r, s} x$ for all units $E_{r, s} \in \triangle_{k}$, which yields $C_{G_{1}}\left(G_{k}\right)=I+\square_{n-k}$. Similarly, we get $C_{G_{1}}\left(I+\square_{k}\right)=I+\square_{n-k}$, $1 \leq k \leq n$. Now it follows from Theorem 2 that any faithful character of $G_{1}$ vanishes on the set $\bigcup_{k=1}^{n}\left(\left(I+\triangle_{k}\right)-\left(I+\square_{n-k}\right)\right)$.

\section{References}

[1] R. J. Blattner, On induced representations, Amer. J. Math. 83 (1961), 79-98.

[2] A. L. Carey and W. Moran, Characters of nilpotent groups, Math. Proc. Cambridge Philos. Soc. 96 (1984), 123-137.

[3] M. Hall, The Theory of Groups, New York, Macmillan, 1969.

[4] R. E. Howe, On representation of finitely generated discrete torsion free nilpotent groups, Pacific J. Math. 73 (1977), 281-305.

[5] E. Kaniuth und R. Lasser, Zum verallgemeinerten Wienerschen Satz über diskrete nilpotente Gruppen der Klasse 3, Math. Z. 163 (1978), 39-55.

[6] E. Płonka, Remarks on characters of discrete nilpotent groups, Math. Ann. 240 (1979), 97-102.

[7] S. Sakai, $C^{*}$-algebras and $W^{*}$-algebras, Springer, 1971.

[8] E. Thoma, Über unitäre Darstellungen abzählbarer Gruppen, Math. Ann. 153 (1964), 111-139.

[9] H. Umegaki, Condional expectation in operator algebras, Tôhoku Math. J. 6 (1954), 1977-1981.

Institute of Mathematics

Silesian Technical University

Kaszubska 23

44-100 Gliwice, Poland

E-mail: eplonka@zeus.polsl.gliwice.pl 